\title{
Une méthode générale d'estimation des paramètres génétiques dans un échantillon sélectionné, avec une application à une sélection sur un indice à trois caractères
}

\author{
L. OLLIVIER et A. DERRIEN (*) \\ I.N.R.A., Station de Génétique quantitative et appliquée \\ Centre de Recherches zootechniques \\ F 78350 Jouy-en-Josas
}

\begin{abstract}
Résumé
L'estimation des paramètres génétiques d'une population, héritabilités et corrélations génétiques, à partir d'un échantillon repose sur de nombreuses hypothèses, qui concernent tant la population elle-même que l'échantillon qu'on en tire. La principale condition à laquelle doit satisfaire l'échantillon est d'être aléatoire, ce qui n'est pas le cas lorsque les individus mesurés, ou leurs parents, sont sélectionnés, situation fréquente chez les animaux d'élevage. Dans ce cas, les estimations obtenues par les techniques classiques de régression et d'analyse de variance peuvent être faussées et des corrections sont nécessaires pour tenir compte de la sélection. Lorsque la sélection se fait sur plusieurs caractères simultanément, combinés par exemple dans un indice de sélection, la correction repose sur la connaissance des variances-covariances des parents sélectionnés relativement à celles de la population non sélectionnée, selon une méthode générale décrite, en particulier, par AITKEN (1934). Cette méthode inclut, comme cas particulier, les résultats de plusieurs travaux récents concernant les effets de la sélection sur un seul caractère. Cependant, la variance d'échantillonnage des estimations corrigées ainsi obtenues a une expression complexe. Un exemple numérique, concernant une sélection sur un indice à 3 caractères chez le Porc, est traité. Il montre un bon accord entre les estimées corrigées pour les effets de la sélection et les estimées tirées de l'échantillon non sélectionné.
\end{abstract}

\section{1. - Introduction}

L'étude génétique des caractères quantitatifs repose sur l'estimation de paramètres permettant d'assigner leur variabilité, lorsqu'on les considère individuellement ou deux à deux, à des facteurs génétiques et de milieu. Les paramètres génétiques essentiels sont les héritabilités $\left(h^{2}\right)$ et les corrélations génétiques $\left(r_{A}\right)$. Ils se déduisent des variances et covariances génétiques additives, celles-ci étant elle-mêmes obtenues

(*) Communication présentée à la deuxième réunion du groupe de Biologie et Génétique des Populations, 28-29 avril 1980 à Gif-sur-Yvette. 
à partir de covariances calculées entre des individus apparentés. Les modèles mathématiques utilisés impliquent des hypothèses assez nombreuses, qu'on peut classer en deux catégories selon qu'elles concernent la population dans son ensemble ou l'échantillon qui en est tiré pour les estimations. La première catégorie d'hypothèses peut se résumer par les conditions de l'équilibre de Hardy-Weinberg. Puisque l'objet de cet article n'est pas d'en discuter, nous supposons ces conditions remplies et nous excluons également, dans la population considérée, les effets du «linkage » et de l'épistasie. Dans la deuxième catégorie d'hypothèses, la principale est le caractère aléatoire de l'échantillon. Nous allons envisager ici les conséquences du non-respect de cette hypothèse dans le cas, assez fréquent chez les animaux d'élevage, où les parents des individus mesurés ont fait l'objet d'une sélection, sur un ou sur plusieurs caractères.

Le tableau 1 donne un aperçu de quelques travaux publiés dans ce domaine depuis 1953. Il s'agit de résultats théoriques, pour la plupart, et d'applications à des espèces animales dans les cas suivants : Drosophile (REEVE, 1953), Souris (RAHNEFEld et al., 1963), Bœuf (RoBerTSON, 1966) et Mouton (BROWN \& TURNer, 1968). Notons aussi que l'étude de VAN VLECK (1968) est basée sur une simulation. Enfin la question a été envisagée dans le cadre plus large des méthodes d'estimation en génétique quantitative par THOMPson (1973 et 1977).

\section{TABLEAU 1}

Etudes des effets de la sélection sur l'estimation des paramètres génétiques

Studies of the effects of selection on the estimation of genetic parameters

\begin{tabular}{l} 
Auteurs \\
\hline
\end{tabular}




\section{2. - Description de la méthode}

L'incidence de la sélection sur l'estimation des paramètres génétiques peut être envisagée d'abord dans un cadre purement statistique. Ce problème fut pour la première fois étudié par K. Pearson en 1902 (cité par AITKEN, 1934). Il considéra en effet le cas de $\mathrm{n}$ variables aléatoires parmi lesquelles $\mathrm{p}$ sont soumises à une sélection de telle sorte que sont modifiées les $p$ moyennes, $p$ variances et $p(p-1) / 2$ covariances. Les effets de cette sélection sur les variances et covariances des $n-p$ variables restantes ont été formulés d'une manière particulièrement simple et condensée par AITKEN (1934) en utilisant la notation matricielle suivante.

La matrice $\mathbf{R}$ des covariances entre $\mathbf{n}$ variables supposées normales centrées réduites ( $R$ est donc une matrice de corrélations) peut être découpée en quatre blocs :

$$
R=\left(\begin{array}{ll}
\mathbf{R}_{p p} & \mathbf{R}_{\mathrm{pq}} \\
\mathbf{R}_{\mathrm{qp}} & \mathbf{R}_{\mathrm{qq}}
\end{array}\right)
$$

Les matrices $R_{p p}$ et $R_{q q}$ sont symétriques et concernent respectivement les $p$ variables soumises à sélection et les $q$ variables restantes $(p+q=n)$. La matrice $R_{p q}$ (dont la transposée est $R_{q p}$ ) donne les corrélations entre les $p$ premières variables et des $q$ dernières deux à deux.

Si une sélection est appliquée aux $p$ variables, la matrice $R_{p p}$ est modifiée et devient, disons, $V_{p p}$. Il en résulte que la matrice $R$ est transformée en une matrice $R^{\prime}$ (qui est une matrice de covariances) telle que :

avec :

$$
R^{\prime}=\left(\begin{array}{ll}
V_{p p} & R_{p q}^{\prime} \\
R^{\prime} & R^{\prime}{ }_{q q}
\end{array}\right)
$$

et :

$$
R_{p q}^{\prime}=V_{p p} R_{p p}^{-1} R_{p q}
$$

$$
R_{q q}^{\prime}=R_{q q}-R_{q p}\left(R_{p p}^{-1}-R_{p p}^{-1} V_{p p} R_{p p}^{-1}\right) R_{p q}
$$

On peut remarquer (J.W. JAMES, communication personnelle) que les relations ( 1 a) et $(1 \mathrm{~b})$, démontrées par AITKEN avec l'hypothèse de multinormalité, avant comme après la sélection, restent vraies sous des hypothèses moins restrictives qui sont (1) la linéarité des régressions des q variables en fonction des p variables, (2) l'homoscédasticité des $\mathrm{q}$ variables autour de leurs régressions en fonction des $\mathrm{p}$ variables. $\mathrm{Si}$ on désigne par $B_{p q}$ la matrice de ces coefficients de régression, la matrice des covariances (des $q$ variables) dues à la régression est $B_{q p} R_{p p} B_{p q}\left(B_{q p}\right.$ étant la transposée de $B_{p q}$ ) et la matrice des covariances des écarts à la régression est $R_{q q 1}-B_{q p} R_{p p} B_{p q-}$ Les matrices $R_{p p}$ et $R_{p q}$ deviennent donc après sélection :

et :

$$
\mathbf{R}_{\mathrm{pq}}^{\prime}=\mathrm{V}_{\mathrm{pp}} \mathrm{B}_{\mathrm{pq}}
$$

$$
R_{q q}^{\prime}=B_{q p} V_{p p} B_{p q}+R_{q q}-B_{q p} R_{p p} B_{p_{q}}
$$

d'où l'on déduit les équations $(1 \mathrm{a})$ et $(1 \mathrm{~b})$ sachant que $\mathrm{B}_{\mathrm{pq}}=\mathrm{R}_{\mathrm{pp}}^{-1} R_{\mathrm{pq}}$. 
A l'inverse, connaissant $R^{\prime}$ et $R_{p p}$ on peut résoudre les équations ci-dessous en $\mathbf{R}_{\mathrm{pq}}$ et $\mathbf{R}_{\mathrm{qq}}$ et obtenir $\mathbf{R}$, c'est-à-dire les covariances dans la population non sélectionnée. On obtient ainsi :

et :

$$
R_{p q}=R_{p p} V_{p p}^{-1} R_{p q}^{\prime}
$$

$$
R_{\mathrm{qq}}=R_{\mathrm{qq}}^{\prime}+R_{\mathrm{qp}}\left(\mathrm{R}_{\mathrm{pp}}^{-1}-\mathrm{R}_{\mathrm{pp}}^{-1} \mathrm{~V}_{\mathrm{pp}} \mathrm{R}_{\mathrm{pp}}^{-1}\right) \mathrm{R}_{\mathrm{pq}}
$$

Connaissant donc $R_{p p}$ et $V_{p p}$ (c'est-à-dire les effets de la sélection sur les variances-covariances des $\mathrm{p}$ variables) on peut calculer d'abord $R_{p q}$ à l'aide de (2a), puis $R_{\mathrm{lq}}$ en reportant dans ( $2 \mathrm{~b}$ ) la valeur $R_{\mathrm{pq}}$ obtenue précédemment.

\section{3. - Applications génétiques}

a) Cas d'un ensemble de caractères mesurés sur l'un des parents et les enfants

Dans cette situation, il est possible d'estimer d'une part les covariances parentenfant et d'autre part, en se limitant à l'analyse de la descendance, les covariances entre demi-germains et, éventuellement, entre germains. Les covariances précédentes ainsi que les variances-covariances des caractères mesurés chez les parents peuvent se disposer selon une matrice analogue à la matrice $R$ définie ci-dessus, où $\mathbf{R}_{\mathrm{pp}}$ concerne le parent mesuré, $R_{p q}$ exprime les covariances parent-enfant et $R_{q q}$ les covariances entre demi-germains. Les variables aléatoires concernées par $\mathbf{R}$ peuvent donc être considérées comme se répartissant en $\mathrm{p}$ aléatoires "parent 》 et $\mathrm{q}$ aléatoires " enfant », en supposant les tirages de la variable «enfant» indépendants entre eux pour un parent donné, ce qui exclut la possibilité de tirer deux germains. Dans certains cas, la relation parent-enfant est envisagée avec l'autre parent (non mesuré) supposé fixé, comme dans la régression «mère-fille intra-père ». Les tirages concernent alors la mère et la fille et $\mathbf{R}_{\mathrm{aq}}$ est dans ce cas une matrice de covariances entre germains. Précisons que, dans cette situation, ces covariances sont égales à la différence entre la covariance entre germains et la covariance entre demi-germains obtenues dans une analyse de variance selon le modèle hiérarchique classique pèremère-descendant, les sommes de carrés (et de produits) étant calculées intra-le père considéré dans la régression. L'application de $(2 \mathrm{a})$ et $(2 \mathrm{~b})$ à l'une ou l'autre de ces situations donne les estimations des covariances parent-enfant, entre demi-germains ou entre germains, "corrigées» pour les effets de la sélection, si on connaît à la fois les covariances $R_{p p}$ dans la population des individus avant sélection et les covariances $\mathrm{V}_{\mathrm{pp}}$ pour les individus sélectionnés.

Le cas particulier de la sélection sur un caractère et des répercussions sur son héritabilité $\left(\mathrm{h}^{2}{ }_{1}\right)$, sur celle d'un deuxième caractère lié au précédent $\left(\mathrm{h}^{2}{ }_{22}\right)$ et sur la corrélation génétique $\left(r_{A}\right)$ entre les deux, donne les relations suivantes en appliquant les équations $(1 \mathrm{~b})$ et en désignant par $h^{\prime}$ et $r_{A}^{\prime}$ les valeurs, modifiées par la sélection, des paramètres obtenus à partir des covariances entre demi-germains :

$$
\mathrm{h}^{\prime 2}{ }_{1}=\mathrm{h}^{2}{ }_{1}\left(1-\mathrm{K} \mathrm{h}^{2}{ }_{1}\right) /\left(1-0,25 \mathrm{~K} \mathrm{~h}^{4}{ }_{1}\right)
$$




$$
\begin{aligned}
& \mathrm{h}^{\prime 2}{ }_{2}=\mathrm{h}^{2}{ }_{2}\left[1-\mathrm{K}\left(\mathrm{r}_{\mathrm{A}} \mathrm{h}_{1}\right)^{2}\right] /\left[1-0,25 \mathrm{~K}\left(\mathrm{r}_{\mathrm{A}} \mathrm{h}_{1} \mathrm{~h}_{2}\right)^{2}\right] \\
& \mathbf{r}_{\mathrm{A}}^{\prime}=\mathrm{r}_{\mathrm{A}} \mathrm{h}_{1} \mathrm{~h}_{2}\left(1-\mathrm{Kh}^{2}{ }_{1}\right) / \mathrm{h}_{1}^{\prime} \mathrm{h}_{2}^{\prime}
\end{aligned}
$$

où,$K$ désigne le facteur de réduction de variance. Dans une sélection d'intensité $\mathrm{i}$ sur une distribution normale, en désignant par $\mathrm{x}$ l'abscisse du point de troncature, on a $K=i(i-x)$. On retrouve ainsi en (3 a) l'expression de la covariance entre demigermains donnée par RAHNEFELD et al. (1963), et en $(3 \mathrm{~b})$ et $(3 \mathrm{c})$ des expressions données par Robertson (1977) et Ponzoni \& James (1978).

\section{b) Cas d'un ensemble de caractères mesurés sur les deux parents et les enfants}

Dans cette situation, l'estimation des paramètres génétiques repose d'une part sur la covariance parent-moyen/enfant et sur les covariances entre germains ou entre demi-germains. La même méthode que ci-dessus est applicable à condition de remplacer l'aléatoire «parent» précédente par le «parent-moyen », $\mathbf{R}_{\mathrm{qq}}$ étant alors une matrice de covariance entre germains. Pour ce qui est des covariances entre demi-germains, il suffit de considérer la sélection appliquée au parent concerné, la sélection appliquée aux mères, par exemple, étant sans incidence sur les covariances entre demi-frères de père. Cela suppose l'homogénéité des variances "mère-intrapère », et c'est d'ailleurs l'une des hypothèses posées dans le modèle d'analyse de variance pour un dispositif hiérarchique.

\section{4. - Exemple numérique}

Pour illustrer la méthode, nous avons retenu un échantillon de 32517 verrats de race Large White et Landrace Français contrôlés en France de 1969 à 1978, et trois caractères mesurés sur chaque individu, la vitesse de croissance (GMQ), le rapport de la consommation d'aliment au gain de poids (IC) et l'épaisseur de lard (LM) mesurée aux ultra-sons sur l'animal vivant (voir Ollivier et al., 1980). Ces données, exprimées en unité d'écart-type, ont été classées selon une hiérarchie élevage-pèremère et soumises à l'analyse de variance et de covariance classique pour un tel dispositif (KEMPTHORNE, 1957). Les paramètres génétiques relatifs aux trois variables ont été estimés à partir des covariances entre demi-germains (intra-élevage), ces covariances étant supposées égales au quart des variances et covariances génétiques additives. Par ailleurs, un sous-échantillon de 11377 verrats, constitué des individus dont le père avait lui-même été contrôlé, a été analysé de la même façon. Les paramètres génétiques relatifs à l'échantillon total et au sous-échantillon qui vient d'être défini sont donnés dans le tableau 2.

A partir du sous-échantillon de 11377 verrats et de leurs 1104 pères, lès covariances parent-descendant ont été estimées selon la méthode décrite par OLIIVIER (1974) ainsi que les variances-covariances des trois caractères chez les 1104 pères. La matrice $R^{\prime}$, définie plus haut, a ainsi été établie (tableau 3). A partir de cette 
matrice et des corrélations phénotypiques du tableau 2 ( $1^{\text {re }}$ ligne), qui constituent la matrice $R_{p p}$, la matrice $R$ est obtenue en appliquant les équations $(2$ a) et ( $2 b$ ). Les résultats sont donnés dans le tableau 3, et leur traduction en héritabilités et corrélations génétiques dans le tableau 4.

\section{TABLEAU 2}

Héritabilités (dans la diagonale), corrélations phénotypiques (au-dessus de la diagonale) et corrélations génétiques (au-dessous de la diagonale), dans l'échantillon complet (1 $1^{\text {re }}$ ligne) et dans le sous-échantillon sélectionné ( $2^{e}$ ligne)

Heritabilities, in the diagonal, phenotypic, above the diagonal, and genetic, below the diagonal, correlations: whole sample on the 1st line, selected sub-sample on the second line

\begin{tabular}{|c|c|c|c|}
\hline & GMQ & IC & LM \\
\hline GMQ $\ldots \ldots \ldots \ldots$ & $\begin{array}{l}0,26 \\
0,27\end{array}$ & $\begin{array}{l}-0,75 \\
-0,76\end{array}$ & $\begin{array}{l}0,03 \\
0,02\end{array}$ \\
\hline IC $\ldots \ldots \ldots \ldots \ldots$ & $\begin{array}{l}-0,72 \\
-0,81\end{array}$ & $\begin{array}{l}0,20 \\
0,16\end{array}$ & $\begin{array}{l}0,15 \\
0,15\end{array}$ \\
\hline LM $\ldots \ldots \ldots \ldots$ & $\begin{array}{l}0,08 \\
0,04\end{array}$ & $\begin{array}{l}0,18 \\
0,11\end{array}$ & $\begin{array}{l}0,39 \\
0,26\end{array}$ \\
\hline
\end{tabular}

GMQ : Gain moyen quotidien.

Average daily gain.

IC : Indice de consommation.

Food conversion.

LM : Epaisseur moyenne de lard.

Average backfat thickness.

Les 3 variables sont exprimées en écart à la moyenne de bande - groupe de contemporains et en unité d'écart-type.

The 3 variables are expressed as standardized deviation from contemporaries.

La sélection réalisée dans le choix des 1104 pères du sous-échantillon est équivalente à une sélection massale d'intensité $\mathrm{i}=1,24$, ce qui correspond à un taux de sélection de 26 p. 100 pour une variable normale, sur un indice linéaire égal à $(\mathrm{GMQ})-0,3(\mathrm{IC})-1,3(\mathrm{LM})$. Il s'agit d'un indice "rétrospectif 》 (voir OllIVIER, 1981, p. 77) légèrement différent de l'indice "voulu » qui est égal à (GMQ) -- 0,6 (IC) - 1,1 (LM). L'incidence de cette sélection sur les covariances entre demi-germains est surtout notable pour le caractère le plus héritable (LM) pour lequel 
$\mathrm{h}^{2}$ est réduit de près de $30 \mathrm{p}$. 100. La correction appliquée donne des valeurs «attendues» très voisines des valeurs observées dans l'échantillon total non sélectionné. En ce qui concerne les régressions parent-descendant, l'effet de la sélection ne peut pas être vérifié directement, puisque seuls les pères sélectionnés (ainsi que des pères non contrôlés) ont des descendants. Le tableau 4 montre cependant que tous les paramètres génétiques sont modifiés par la sélection dans le même sens avec les deux estimateurs considérés et on note, en particulier, l'inversion du signe de la corrélation GMQ-LM dans les deux cas.

\section{TABleau 3}

Matrice $R^{\prime}$ des covariances estimées dans l'échantillon sélectionné ( $1^{\text {re }}$ ligne) et matrice $R$ des covariances corrigées pour les effets de la sélection $\left(2^{e}\right.$ ligne)

Ist line : covariance matrix $R^{\prime}$ from the selected sub-sample; $2 d$ line : covariance matrix $R$, corrected for the effects of selection

\begin{tabular}{|c|c|c|c|c|c|c|c|}
\hline & & \multicolumn{3}{|c|}{$\begin{array}{c}\text { Parent }(\mathrm{P}) \\
\text { Parent }\end{array}$} & \multicolumn{3}{|c|}{$\begin{array}{c}\text { Descendant (D) } \\
\text { Offspring }\end{array}$} \\
\hline & & GMQ & IC & LM & GMQ & IC & LM \\
\hline & $\mathbf{P}$ & & & & & & \\
\hline GMQ & $\ldots \ldots \ldots$ & $\begin{array}{l}0,697 \\
1\end{array}$ & $\begin{array}{l}-0,408 \\
-0,754\end{array}$ & $\begin{array}{l}0,152 \\
0,033\end{array}$ & $\begin{array}{r}0,077 \\
-0,108\end{array}$ & $\begin{array}{l}-0,063 \\
-0,065\end{array}$ & $\begin{array}{r}0,003 \\
-0,017\end{array}$ \\
\hline IC .. & $\ldots \ldots \ldots$ & & $\begin{array}{l}0,520 \\
1\end{array}$ & $\begin{array}{r}-0,066 \\
0,148\end{array}$ & $\begin{array}{l}-0,063 \\
-0,108\end{array}$ & $\begin{array}{l}0,051 \\
0,110\end{array}$ & $\begin{array}{l}0,010 \\
0,052\end{array}$ \\
\hline LM & . & & & $\begin{array}{l}0,535 \\
1\end{array}$ & $\begin{array}{r}0,007 \\
-0,045\end{array}$ & $\begin{array}{l}0,018 \\
0,099\end{array}$ & $\begin{array}{l}0,101 \\
0,216\end{array}$ \\
\hline & $\mathrm{D}$ & & & & & & \\
\hline GMQ & & & & & $\begin{array}{l}0,067 \\
0,070\end{array}$ & $\begin{array}{l}-0,042 \\
-0,047\end{array}$ & $\begin{array}{r}0,003 \\
-0,004\end{array}$ \\
\hline IC & $\ldots \ldots \ldots$ & & & & & $\begin{array}{l}0,040 \\
0,048\end{array}$ & $\begin{array}{l}0,005 \\
0,018\end{array}$ \\
\hline LM & $\cdots$ & & & & & & $\begin{array}{l}0,066 \\
0,095\end{array}$ \\
\hline
\end{tabular}




\section{TABLEAU 4}

Héritabilités $\left(h^{2}\right)$ et corrélations génétiques $\left(r_{A}\right)$ estimées dans l'échantillon complet et dans le sous-échantillon sélectionné

Heritabilities and genetic correlations estimated in the selected sub-sample and in the whole sample

\begin{tabular}{|c|c|c|c|}
\hline \multirow{2}{*}{$\begin{array}{l}\text { Estimateur } \\
\text { Estimator }\end{array}$} & \multicolumn{2}{|c|}{$\begin{array}{l}\text { Sous-échantillon sélectionné } \\
\text { Sub-sample }\end{array}$} & \multirow{2}{*}{$\begin{array}{l}\text { Echantillon total } \\
\text { Whole sample }\end{array}$} \\
\hline & $\begin{array}{l}\text { Sans correction } \\
\text { Uncorrected }\end{array}$ & $\begin{array}{l}\text { Après correction } \\
\text { Corrected }\end{array}$ & \\
\hline (1) $\mathrm{h}^{2}$ Basé sur Cov & & & \\
\hline 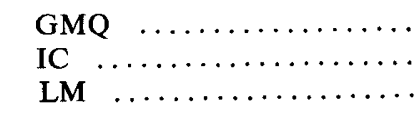 & $\begin{array}{l}0,27 \pm 0,04 \\
0,16 \pm 0,03 \\
0,26 \pm 0,04\end{array}$ & $\begin{array}{l}0,28 \\
0,19 \\
0,38\end{array}$ & $\begin{array}{l}0,26 \pm 0,02 \\
0,20 \pm 0,02 \\
0,39 \pm 0,02\end{array}$ \\
\hline $\begin{array}{l}\text { (2) } \mathrm{r}_{\mathrm{A}} \\
\text { GMQ-IC } \ldots \ldots \ldots \ldots \ldots \ldots \ldots \ldots \ldots \ldots \ldots \ldots \ldots \\
\text { GMQ-LM } \ldots \ldots \ldots \ldots \ldots \ldots \ldots\end{array}$ & $\begin{array}{r}-0,81 \pm 0,05 \\
0,04 \pm 0,10 \\
0,11 \pm 0,12\end{array}$ & $\begin{array}{r}-0,81 \\
-0,05 \\
0,27\end{array}$ & $\begin{array}{r}-0,72 \pm 0,03 \\
0,08 \pm 0,05 \\
0,18 \pm 0,06\end{array}$ \\
\hline (3) $\mathrm{h}^{2}$ Basé sur b & & & \\
\hline $\begin{array}{l}\text { GMQ } \quad \ldots \ldots \ldots \ldots \ldots \ldots \ldots \\
\text { IC } \ldots \ldots \ldots \ldots \ldots \ldots \ldots \\
\text { LM } \ldots \ldots \ldots \ldots \ldots \ldots\end{array}$ & $\begin{array}{l}0,22 \pm 0,04 \\
0,20 \pm 0,04 \\
0,38 \pm 0,04\end{array}$ & $\begin{array}{l}0,22 \\
0,22 \\
0,43\end{array}$ & $\overline{-}$ \\
\hline (4) $r_{A}$ & & & \\
\hline $\begin{array}{l}\text { GMQ-IC } \ldots \ldots \ldots \ldots \ldots \ldots \\
\text { GMQ-LM } \ldots \ldots \ldots \ldots \ldots \ldots \\
\text { IC-LM } \ldots \ldots \ldots \ldots \ldots\end{array}$ & $\begin{array}{r}-0,72 \pm 0,06 \\
0,05 \pm 0,09 \\
0,19 \pm 0,06\end{array}$ & $\begin{array}{r}-0,77 \\
-0,18 \\
0,47\end{array}$ & - \\
\hline
\end{tabular}

Cov : Covariance entre demi-germains. Half-sib covariance.

(1) $h^{2}=4$ Cov.

(2) $r_{\mathrm{A}}$ (entre caractères 1 et 2$)=\operatorname{Cov}_{12} /\left(\operatorname{Cov}_{1} \operatorname{Cov}_{2}\right)^{0,5}$.

b : Régression parent-enfant.

(3) $h^{2}=2 b$.

(4) $r_{\Delta}= \pm\left(b_{12} b_{12} / b_{11} b_{22}\right)^{0,5}\left(b_{12}\right.$ régression du caractère 1 chez le descendant en fonction du caractère 2 chez le parent, etc.). 


\section{5. - Discussion et conclusions}

Les effets de la sélection de l'échantillon sur les paramètres génétiques — équations (1) - sont clairement établis dans le cas de la sélection sur un seul caractère. Comme le montrent les équations (3) les variances et covariances génétiques déduites des covariances entre demi-germains sont modifiées dans le sens du changement de la variance phénotypique, et ce d'autant plus que l'héritabilité du caractère sélectionné est plus élevée. On sait par ailleurs que la sélection des parents sur un seul caractère n'affecte pas la régression parent-enfant pour ce caractère. La généralisation de ces principes au cas de $\mathbf{n}$ caractères sélectionnés ne paraît pas possible, le résultat dépendant d'un trop grand nombre de paramètres. La simulation de VAN VLECK (1968), réalisée pour 2 caractères, est difficile à étendre au-delà, car le nombre de paramètres génétiques à faire varier pour $n$ caractères est $n(n+1) / 2$, et par ailleurs les modalités de la sélection elle-même deviennent trop nombreuses pour qu'elles puissent toutes être envisagées. Chaque cas doit donc être traité séparément. La méthode ci-dessus, qui n'est pas nouvelle, permet de le faire assez simplement quel que soit le nombre $n$ de variables.

L'opération inverse de la précédente, qui est l' «élimination » - sur la base des équations (2) - du biais dû à la sélection, est illustrée numériquement au tableau 4 et la méthode utilisée, si elle ne permet pas de retrouver exactement les paramètres de la population dans son ensemble, donne cependant des estimations qui s'en rapprochent. Face à un échantillon sélectionné, le généticien « quantitatif 》 adopte généralement l'une des trois attitudes suivantes, comme le remarque THOMPSON (1979) : (1) ignorer la sélection, (2) corriger pour la sélection, (3) appliquer des méthodes d'estimation utilisant toute l'information disponible y compris celle relative à la sélection (Curnow, 1961). C'est l'option (2) que nous avons retenue dans ce qui précède. Comme nous l'avons vu, l'estimation du biais est assez simple, mais la méthode ne permet pas de calculer facilement la variance des estimateurs ( 2 a) et $(2 \mathrm{~b})$. Il suffit de se reporter à l'article de DE METs \& HALPERIN (1977), qui envisagent pourtant un cas relativement simple, pour se rendre compte de la complexité des expressions de ces variances. $\mathrm{La}$ méthode du maximum de vraisemblance est sans doute la solution la plus satisfaisante (Thompson, 1977), car elle résout à la fois le problème de l'estimation combinée, qui se pose chaque fois que le nombre des covariances entre apparentés est supérieur à celui des paramètres à estimer, et le problème de la variance de l'estimateur. Mais, en contrepartie, on se heurte à la complexité du calcul de l'estimateur lui-même, surtout lorsque le dispositif expérimental est déséquilibré.

Rappelons enfin que la sélection n'est pas toujours un inconvénient dans l'estimation des paramètres génétiques. Comme l'a souligné REeve (1953), une sélection délibérément pratiquée, éventuellement accompagnée d'homogamie, permet, en augmentant la variance des parents, d'obtenir des estimations plus précises et c'est cet avantage que les expériences de sélection bi-directionnelles permettent d'exploiter.

Reçu pour publication en juillet 1981. 


\title{
Remerciements
}

Nous remercions pour leurs remarques et suggestions les lecteurs de cet article, J.W. JAMES (Université de Sydney, Australie) et R. Rouvier (I.N.R.A., Toulouse).

\author{
Summary \\ A general method of estimating genetic parameters in a selected sample, \\ with an application to a three-trait index selection
}

The estimation of genetic parameters rests on several hypotheses, concerning either the population considered itself or the sample drawn from it. In particular, random sampling has to be assumed, which is not the case when the individuals measured, or their parents, are selected, a frequent situation in farm animals. In this case, the usual estimates from regression and analysis of varianice may be biassed. When selection is based on several traits, which for instance are combined into an index, the bias may be derived from the knowledge of the phenotypic variance-covariance matrix of the selected parents, according to a general method described, in particular, by AITKEN (1934). This method includes, as particular cases, several more recent results concerning the bias due to selection on one character. However, the sampling variances of the estimates so obtained have complex expressions. A numerical illustration is given, which concerns a three-trait index selection in the pig. The results show a good agreement between the estimates corrected for the bias due to selection and the estimates drawn from the unselected sample.

\section{Références bibliographiques}

AItken A.C., 1934. Note on selection from a multivariate normal population. Proc. Edinburgh Math. Soc., 4, 106-110.

Brown G.H., TURNer H.N., 1968. Response to selection in Australian Merino sheep. II. - Estimates of phenotypic and genetic parameters for some production traits on Merino ewes and an analysis of the possible effects of selection on them. Aust. J. agric. Res., 19, 303-322.

CURNOW R.N., 1961. The estimation of repeatability and heritability from records subject to culling. Biometrics, 17, 553-566.

De Mets D., Halperin M., Estimation of a simple regression coefficient in samples arising from a sub-sampling procedure. Biometrics, 33, 47-56.

KempthoRne O., 1957. An Introduction to Genetic Statistics. John Wiley and Sons, New York.

Ollivier L., 1974. La régression parent-descendant dans le cas de descendances subdivisées en familles de taille inégale. Biometrics, 30, 59-66.

Ollivier L., 1981. Eléments de Génétique quantitative. Masson, Paris.

Olimier L., Derrien A., Molénat M., 1980. Paramètres génétiques des verrats Large White et Landrace français soumis au contrôle individuel de 1969 à 1978. Analyse préliminaire. Techni-Porc, 3 (1), 7-12. 
Ponzoni R.W., James J.W., 1978. Possible biases in heritability estimates from intraclass correlations. Theor. appl. Genet., 53, 25-27.

Rahnefeld G.W., Boylan W.J., Comstock R.E., Singh M., 1963. Mass selection for postweaning growth in mice. Genetics, 48, 1567-1583.

REEVE E.C.R., 1953. Studies in quantitative inheritance. III. - Heritability and genetic correlation in progeny tests using different mating systems. J. Genet., 51, 520-542.

Robertson A., 1966. A mathematical model of the culling process in dairy cattle. Anim. Prod., 8, 95-108.

Robertson A., 1977. The effect of selection on the estimation of genetic parameters. Z. Tierzüchtg. Züchtgsbiol., 94, 131-135.

THOMPson R., 1973. The estimation of variance and covariance components with an application when records are subject to culling. Biometrics, 29, 527-550.

Thompson R., 1977. Estimation of quantitative genetic parameters. In Pollak E., KempTHORNe O., Bailey T.B. Jr., Proceedings of the International Conference on Quantitative Genetics, 639-657. The Iowa State University Press, Ames, Iowa.

Гномтson R., 1979. Sire evaluation. Biometrics, 35, 339-353.

VAN Vleck L.D., 1968. Selection bias in estimation of the genetic correlation. Biometrics, 24, 951-964. 